\title{
Transduction Complicates the Detection of Conjugative Ability in Lysogenic Salmonella Strains
}

\author{
By H. WILLIAMS SMITH* AND MARGARET A. LOVELL \\ Houghton Poultry Research Station, Houghton, Huntingdon, Cambridgeshire PE17 2DA, UK
}

(Received 31 December 1984 ; revised 18 March 1985)

\begin{abstract}
When lysogenic salmonella strains were examined for conjugative ability in tri-parental crosses, false positive results were sometimes obtained because phage carried by the lysogenic strains multiplied on the intermediate salmonella recipient strain and then transduced its streptomycin/ sulphonamide resistance plasmid to the final salmonella recipient strain. Back transfer of the plasmid to the lysogenic strains was also detected.
\end{abstract}

\section{INTRODUCTION}

As an essential preliminary to a search for salmonella strains possessing conjugative plasmids encoding virulence, strains with conjugative ability $\left(\mathrm{Tra}^{+}\right)$were detected by their ability to mobilize streptomycin/sulphonamide resistance plasmids $(\mathrm{SmSu})$ from a $\mathrm{Tra}^{-}$Salmonella typhimurium strain to another $S$. typhimurium strain. The procedure employed was essentially that of Anderson (1965) and Anderson \& Lewis (1965) except that all three strains in each triparental cross were salmonellae. In many of the crosses the organisms of the final recipient strain that had acquired the $\mathrm{SmSu}$ plasmid of the intermediate recipient strain had, in addition, become lysogenic. Because negative results were obtained when some of these crosses were repeated, this time using Escherichia coli $\mathrm{K} 12$ as the final recipient, it appeared that phage might have been implicated in the plasmid transfer. Further investigations confirmed that this was indeed so; the results of these investigations are reported in this paper.

\section{METHODS}

Bacterial strains. The salmonella strains examined for conjugative ability did not possess transferable properties such as antibiotic resistance and colicin production; they had been isolated from unrelated infections of man or domestic animals. Unless stated, the intermediate recipient strain (IR), which possessed a non-conjugative streptomycin/sulphonamide resistance plasmid $(\mathrm{SmSu})$, and the final recipient strain (FR) employed in the triparental crosses were of Salmonella typhimurium phage type 36 and were obtained from Professor E. S. Anderson (formerly Director of the Enteric Reference Laboratory, Colindale Avenue, London NW9, UK); IR and FR were non-lysogenic. The FR strain was used in these crosses as a spontaneous mutant resistant to sodium nalidixate, spectinomycin and rifampicin ( $\mathrm{Nal}^{r} \mathrm{Spc}^{\mathrm{r}} \mathrm{Rif}^{\mathrm{r}}$ ). On some occasions a Nalr lac mutant of an E. coli $\mathrm{K} 12(\mathrm{SmSu})$ strain was used as intermediate recipient and $\mathrm{Nal}^{r} \mathrm{Spc}^{r}$ and $\mathrm{Amp}^{\mathrm{r}}$ (ampicillin resistant) mutants of $E$. coli $\mathrm{K} 12$ as final recipients. The purpose of using a variety of mutants was to aid the selection of final recipient organisms that had acquired the SmSu plasmids.

Culture media and cultural conditions. Broth cultures consisted of organisms grown in $10 \mathrm{ml}$ nutrient broth, Oxoid no. 2 (CM67). The nutrient agar employed was Difco Tryptose Agar (B66) and the MacConkey's agar was from Oxoid (CM7); Sensitest Agar (Oxoid, CM409) was used for antibiotic-sensitivity tests. All cultures were incubated at $37^{\circ} \mathrm{C}$ for $24 \mathrm{~h}$; fluid cultures, except those used in transfer experiments, were shaken in a water bath (approx. 100 r.p.m.).

Antibiotic sensitivity tests. These were performed by the method of Smith (1970).

Detection of strains with transferring ability. Nutrient broth $(10 \mathrm{ml})$ was inoculated with one drop $(0.03 \mathrm{ml})$ of a broth culture of the strain under test (transferring strain) and nine drops of a broth culture of the $S$. typhimurium intermediate recipient strain $[\mathrm{IR}(\mathrm{SmSu})]$ and incubated. This intermediate mating mixture was left at room temperature for $24 \mathrm{~h}$ to facilitate conjugation by temperature-sensitive plasmids and $1.0 \mathrm{ml}$ was then inoculated 
into $10 \mathrm{ml}$ fresh broth with a similar volume of a broth culture of the $\mathrm{Nal}^{\mathrm{r}} \operatorname{Spc}^{\mathrm{r}} \operatorname{Rif}^{\mathrm{r}} S$. typhimurium final recipient strain (FR) and incubated. This, the final mating mixture, was left at room temperature for $24 \mathrm{~h}$ and $0.5 \mathrm{ml}$ inoculated onto a plate of MacConkey's agar containing $20 \mu \mathrm{g}$ sodium nalidixate $\mathrm{ml}^{-1}$ and $30 \mu \mathrm{g} \mathrm{ml}^{-1}$ each of spectinomycin and streptomycin (the selection medium) and the plate incubated. If colonies that grew on this plate were subsequently found to be resistant to rifampicin and sulphathiazole it was accepted that transfer of the SmSu plasmid had taken place. In crosses employing the $E$. coli $\mathrm{K} 12$ intermediate and/or final recipient strains the antibiotics used in the selection medium and in the sensitivity tests performed on colonies that grew on it were those, too, that facilitated the identification of SmSu plasmid transfer.

Identification of lysogenic strains, purification and propagation of phages, isolation of phage-resistant bacterial mutants, cross-resistance tests, and bacterial and phage counts. The techniques employed were essentially those of Smith \& Huggins (1982) and Smith et al. (1983). When necessary, all phage preparations were rendered free of viable bacteria by holding at $58^{\circ} \mathrm{C}$ for $40 \mathrm{~min}$ or by filtering through a Millipore filter (pore diameter $450 \mathrm{~nm}$ ); their sterility was confirmed by incubating $0.5 \mathrm{ml}$ in nutrient broth.

\section{RESULTS AND DISCUSSION}

In tri-parental crosses 16 of 41 Salmonella typhimurium strains and 11 of 135 strains of other salmonella serotypes transferred the $\mathrm{SmSu}$ plasmid from the $S$. typhimurium intermediate recipient strain [IR(SmSu)] to the $S$. typhimurium final recipient strain (FR). When E. coli $\mathrm{K} 12$ was the final recipient and $\mathrm{IR}(\mathrm{SmSu})$ or a $\mathrm{K} 12(\mathrm{SmSu})$ strain was the intermediate recipient only one of the $16 S$. typhimurium strains and 3 of the 11 strains of other serotypes transferred the $\mathrm{SmSu}$ plasmids. The examination of an FR(SmSu) isolate from each of the original tri-parental crosses involving these 23 strains that had transmitted the plasmids to FR but had failed to transmit them to $\mathrm{K} 12$ revealed that 19 of them were now lysogenized with phages active on FR. The 23 tri-parental crosses were repeated using intermediate mating mixtures which, after incubation, were freed of viable bacteria by filtration or heat-treatment. Transfer of the SmSu plasmid to FR still occurred in the case of 14 of the strains. The numbers of FR(SmSu) organisms in most of the final mating mixtures varied from 150 to $500 \mathrm{ml}^{-1}$, suggesting a transfer rate about 10 times greater than that observed when the strains were examined by the standard method. Transfer by some of the strains, though, occurred at a lower rate and occasionally a strain had to be tested more than once before a positive result was obtained. The nine strains that failed to transfer by the modified method were retested but this time the broth cultures of the transferring strains and not of the intermediate mating mixtures were filtered or heat-treated. Transfer was demonstrated by six of them. The $12 \mathrm{~S}$. typhimurium strains that transferred the SmSu plasmid by one of the modified methods were of phage types 12a (two strains), 14 (two strains), 29, 56, 104 (two strains), 134 and 180; two were untypable. The eight strains of other serotypes that did this were $S$. derby, $S$. heidelberg, $S$. muenster, $S$. poona (2), $S$. saint-paul, $S$. seftenberg and $S$. worthington. The phages with which the $12 S$. typhimurium strains were lysogenized were propagated on FR and then examined to see whether they could transduce the SmSu plasmid of IR(SmSu) to FR. All did. Resistant mutants of FR to each of the 12 phages were obtained and the mutants then tested for susceptibility to all 12 phages. These cross-resistance tests revealed that the phages were of five different kinds. The 12 resistant mutants, all of which were lysogenic, were then grown with IR(SmSu) as in the first part of a triparental cross. These intermediate mating mixtures were cultured on selection medium and all except one of them were found to contain $\mathrm{FR}(\mathrm{SmSu})$ organisms, $15-50 \mathrm{ml}^{-1}$. Similar results were obtained when three $\mathrm{Nal}^{\mathrm{r}} \mathrm{Spc}^{\mathrm{r}} \mathrm{Rif}^{\mathrm{r}}$ lysogenic $S$. typhimurium strains were grown with six $\mathrm{Sm}^{\mathrm{r}} \mathrm{Su}^{\mathrm{r}}$ S. typhimurium strains; $\mathrm{SmSu}$ transfer was never detected from any of the $\operatorname{six}^{\mathrm{Sm}} \mathrm{Sm}^{\mathrm{r}} \mathrm{Su}^{\mathrm{r}}$ strains to FR or to E. coli K12.

Table 1 summarizes the results of estimating the amount of phage in the intermediate mating mixtures and the amount of $\mathrm{FR}(\mathrm{SmSu})$ organisms in the final mating mixtures of tri-parental crosses in which the inocula of the intermediate mating mixtures consisted of different amounts of strain 7 , a $S$. typhimurium strain of phage type 29 , and IR(SmSu). SmSu transfer was only detected in those crosses in which the amount of $\operatorname{IR}(\mathrm{SmSu})$ in the inoculum of the intermediate mixture was the same as or greater than that of strain 7 , the degree of transfer being directly proportional to the amount of phage generated in the intermediate mating mixture. 
Table 1. Relationship between bacterial and phage content of mating mixtures and SmSu plasmid transfer in tri-parental crosses

Strain 7, transferring strain; IR, intermediate recipient; FR, final recipient. The broth culture of strain 7 used to inoculate the intermediate mating mixture contained $30 \times 10^{2}$ viable phage particles $\mathrm{ml}^{-1}$. The results shown are from a single experiment.

$\begin{array}{ccr}\begin{array}{c}\text { Ratio of no. of strain } 7 \\ \text { organisms to no. of IR(SmSu) } \\ \text { organisms in inoculum of } \\ \text { intermediate mating } \\ \text { mixture }\end{array} & \begin{array}{c}\text { No. of viable phage particles } \\ \mathrm{ml}^{-1} \text { in intermediate mating }\end{array} & \begin{array}{c}\text { No. of FR } \\ \text { organisms } \\ \text { minal mating }\end{array} \\ 100: 1 & 5 \times 10^{+} & 0 \\ 10: 1 & 3 \times 10^{7} & 15 \\ 1: 1 & 8 \times 10^{8} & 300 \\ 1: 10 & 5 \times 10^{9} & 100 \\ 1: 100 & 1 \times 10^{9} & \end{array}$

To gain an impression of the extent to which transduction might complicate tri-parental crosses of organisms other than salmonellae, $27 \mathrm{E}$. coli strains shown by Smith \& Linggood (1970) to transfer SmSu plasmids from an E. coli $\mathrm{K} 12(\mathrm{SmSu})$ strain to another K12 strain were examined for lysogeny to $\mathrm{K} 12$. Only three were lysogenic. In triparental crosses in which the intermediate mating mixtures were heat-treated or filtered the three failed to transfer the $\mathrm{SmSu}$ plasmid from the K12(SmSu) strain. Another eight $E$. coli strains lysogenized with phages active on $\mathrm{K} 12$ were also examined by this method; SmSu plasmid transfer was not detected. These results imply that phage would rarely affect the reliability of triparental crosses for identifying $E$. coli strains with conjugative or non-conjugative plasmids. However, it would be prudent when examining any bacterial strain for these properties to include, if possible, in the tri-parental cross, as intermediate or final recipient, a strain of a genus different from that of the strain under investigation. This conforms to the original description of the tri-parental cross by Anderson (1965) and Anderson \& Lewis (1965). The fact that some lysogenic salmonella organisms acquired the $\mathrm{SmSu}$ plasmid when grown in mixed culture with $\mathrm{SmSu}^{+}$strains suggests that incorrect results might be obtained if lysogenic salmonella strains are used as recipients in direct tests for identifying strains with conjugative plasmids encoding characteristics such as antibiotic resistance. These experiments also suggest that phage might be more important in the ecology of salmonella plasmids than is generally thought.

We are grateful to Professor E. S. Anderson, Professor P. M. Biggs and Mrs Shirley Pilcher for help and assistance in a variety of ways.

\section{REFERENCES}

Anderson, E. S. (1965). A rapid screening test for transfer factors in drug-sensitive Enterobacteriaceae. Nature, London 208, 1016-1017.

Anderson, E. S. \& Lewis, M. J. (1965). Characterization of a transfer factor associated with drug resistance in Salmonella typhimurium. Nature, London 208, 843-849.

Smith, H. Williams (1970). The transfer of antibiotic resistance between strains of enterobacteria in chickens, calves and pigs. Journal of Medical Microbiology 3, 165-180.

Smith, H. Williams \& Huggins, M. B. (1982).
Successful treatment of experimental Escherichia coli infections in mice using phage: its general superiority over antibiotics. Journal of General Microbiology 128, 307-318.

Smith, H. Williams \& Linggood, M. A. (1970). Transfer factors in Escherichia coli with particular regard to their incidence in enteropathogenic strains. Journal of General Microbiology 62, 287-299.

Smith, H. Williams, Green, P. \& Parsell, Z. (1983). Vero cell toxins in Escherichia coli and related bacteria : transfer by phage and conjugation. Journal of General Microbiology 129, 3121-3137. 